\title{
T1T2 Mapping and Texture Analysis of Cine and T2- weighted Magnetic Resonance Imaging to Detect Myocardial Tissue Changes Associated with Chronic Kidney Disease
}

\section{Yasuo Amano ( $\nabla$ yas-amano@nifty.com )}

Surugadai Nihon University Hospital

\section{Yuko Omori}

Surugadai Nihon University Hospital

\section{Maki Amano}

Surugadai Nihon University Hospital

Chisato Ando

Surugadai Nihon University Hospital

Naoki Shinoda

Surugadai Nihon University Hospital

Yasuyuki Suzuki

Surugadai Nihon University Hospital

Naoya Matsumoto

Surugadai Nihon University Hospital

\section{Research Article}

Keywords: Myocardial tissue, chronic kidney disease (CKD), heart failure, serious ventricular arrhythmia

Posted Date: August 20th, 2021

DOl: https://doi.org/10.21203/rs.3.rs-770490/v1

License: (c) (1) This work is licensed under a Creative Commons Attribution 4.0 International License. Read Full License 


\section{Abstract}

Myocardial tissue changes associated with chronic kidney disease (CKD) may lead to heart failure, serious ventricular arrhythmia, and sudden cardiac death. Here, we sought to determine usefulness of T1T2 mapping and texture analysis of T2-weighted short inversion time inversion recovery (STIR) and cine imaging to detect myocardial changes associated with CKD. We examined 34 patients with CKD and 10 controls using a $1.5 \mathrm{~T}$ system. T1 and T2 values of the septal myocardium were significantly greater in patients than in controls ( $P=0.021$ for T1 and $P<0.01$ for T2). The open-access texture analysis software, including features reduction methods, selected one particular feature for T2-weighted STIR and another for cine imaging: vertical fraction for STIR and horizontal short-run emphasis for cine imaging, which were significantly lower in the patients with CKD than in the controls ( $P<0.01$ for both). Texture analysis was not significantly better than T1T2 mapping in detecting the myocardial tissue changes, whereas STIR suffered from image artifacts in 9 patients. In conclusion, additional T1T2 mapping or postprocessing texture analysis may be feasible for detection of myocardial tissue changes associated with CKD.

\section{Introduction}

Cardiovascular complications, including ischemic cardiomyopathy and ventricular arrhythmias, are the most frequent causes of death in patients with chronic kidney disease (CKD), especially in those under dialysis $^{1-4}$. Myocardial hypertrophy, interstitial edema and fibrosis are observed histologically, and these may lead to the cardiac dysfunction and arrhythmia ${ }^{4,5}$. Although endomyocardial biopsy is the reference standard for diagnosis of any cardiomyopathy, it is invasive and can suffer from sampling error. Indeed, the biopsy is rarely performed in patients with CKD.

Magnetic resonance imaging (MRI) is a noninvasive tool useful for identifying cardiomyopathy related to serious cardiac events in a wide variety of myocardial diseases. Late gadolinium enhancement (LGE) MRI and extracellular volume measurement using pre- and postcontrast T1 mapping are powerful methods for this purpose, including uremic cardiomyopathy ${ }^{6-8}$; however, gadolinium-based contrast agents are contraindicated those with CKD at an advanced stage. T1 and T2 mapping MRI quantify the myocardial tissue changes associated with CKD without use of gadolinium ${ }^{8-13}$. Either $\mathrm{T} 1$ or $\mathrm{T} 2$ value is prolonged in CKD, and T2 mapping is useful for differentiating the cardiomyopathy induced by CKD from the normal myocardium and hypertrophied myocardium in patients with hypertensive cardiomyopathy or hypertrophic cardiomyopathy ${ }^{10,12}$. However, there are some disadvantages for T1T2 mapping: the need of additional sequences, absence of standardization for imaging sequences, and limited coverage of the left ventricle. Texture analysis is a postprocessing, quantitative analysis of digital medical images, including MRI. The merit of texture analysis is its postprocessing to be applied to any digital imaging database. To date, texture analysis has been applied to cine, LGE, and T2-weighted short inversion time inversion

recovery (STIR) to detect both ischemic and nonischemic cardiomyopathy ${ }^{14-16}$, but there is only one report applying texture analysis to cardiac MRI of $\mathrm{CKD}^{17}$. Here, we sought to determine whether texture 
analysis of T2-weighted STIR and cine images can detect myocardial tissue changes associated with CKD. We hypothesized that texture analysis could identify the myocardial abnormalities in patients with CKD and would allow for omission of additional T1T2 mapping sequences.

\section{Results (Tables ) \\ Participants.}

T1 and T2 values of the septal myocardium were measured successfully in all of the 34 patients with CKD. By contrast, texture analysis of the entire myocardium was achieved in 24 of the 34 patients; flow or respiratory artifacts interfered with the analysis of T2-weighted STIR images in 9 patients and that of cine images in 4 patients with CKD. Accordingly, 24 CKD patients were recruited for the further analyses. In 10 controls, both T1T2 mapping and texture analysis of T2-weighted STIR and cine images were completed.

\section{T1T2 Mapping.}

T1 $(P=0.021)$ and T2 $(P<0.01)$ values of the myocardium were significantly greater in the patients than in the controls (Figs. $1 \mathrm{~A}, \mathrm{C}$ ). The area under the curve (AUC) was 0.741 for T1 with the cutoff value of $1055 \mathrm{~ms}$ and 0.760 for T2 with a cutoff value of $52.5 \mathrm{~ms}$ on a receiver operating curve (ROC) analysis (Fig. 2).

\section{Texture Analysis.}

The texture analysis software including 4 features reduction methods selected vertical fraction (Vetrl Fraction) for STIR and horizontal short-run emphasis (Horzl shREmp) for cine imaging to discriminate the patients from controls. The Vetrl Fraction of STIR and Horzl shREmp of cine images were significantly smaller in patients with CKD than in controls ( $P<0.01$ for both; Figs. $1 \mathrm{~B}, \mathrm{D})$. An AUC was 0.883 for Vetrl Fraction with a cutoff value of 0.517 and 0.883 for Horzl shREmp with a cutoff value of 0.599 (Fig. 2).

\section{Comparison between T1T2 Mapping and Texture Analysis.}

The specificity was 1.00 for myocardial T2 values and Vetrl Fraction of STIR to discriminate between the CKD patients and controls. Although the AUC of the two texture features noted above was greater than that of $\mathrm{T} 1$ and $\mathrm{T} 1$ values, the differences were not significant $(P>0.05$; Fig. 2$)$.

\section{Discussion}

This study demonstrated that both T1T2 mapping and texture analysis for T2-weighted STIR and cine MRI discriminated the patients with CKD from controls. Texture analysis was not significantly better than 
T1T2 mapping for this purpose, whereas STIR suffered from image artifacts in 9 patients. Therefore, T1T2 values and the two texture features, including Vetrl Fraction of STIR and Horzl shREmp of cine imaging, may reflect myocardial tissue changes associated with CKD.

Both $\mathrm{T} 1$ and $\mathrm{T} 2$ values of the septal myocardium were prolonged in the patients with CKD compared with controls. Previous studies have reported similar results ${ }^{8-10,12,13}$, and these may be related to myocardial fibrosis, cardiomyocytes swelling, and interstitial edema ${ }^{4,18,19}$. Some underlying diseases of CKD, such as hypertension and diabetes mellitus, could affect the myocardial T1 or T2 values ${ }^{12,20,21}$. One previous study has shown that $\mathrm{T} 2$ values in the patients with CKD and myocardial hypertrophy are greater than those of hypertrophied myocardium in patients with hypertensive cardiomyopathy ${ }^{12}$. This finding indicates that the greater T2 values may mainly reflect cardiomyopathy induced by CKD, but not that associated with underlying diseases. The tissue water contents and their interaction with micromolecular components, extracellular expansion or vascular dilatation and myocardial fibrosis may be related to the greater T2 values of the septal myocardium ${ }^{4,18,22}$.

In the present study using texture analysis, Vetrl Fraction of STIR and Horzl shREmp of cine imaging were significantly lower in the patients with CKD than in the controls. The lower Vetrl Fraction means the reduced percentage of the image pixels that are in any of the runs considered for the matrix computing in the vertical direction, while the lower Horzl shREmp represents decrease in the proportion of runs occurring in the images that have short length in the horizontal direction ${ }^{23,24}$. It is difficult to translate these results into histological findings directly, but unexpected homogeneity of the myocardial tissue contents is indicated in the patients with CKD. The AUC of the two texture features noted above was greater than that of T1 and T2 values, but the differences were not significant. The specificity was 1.00 for myocardial T2 values and Vetrl Fraction of STIR, whereas texture analysis for STIR was interfered with by image artifacts in $9(26.4 \%)$ of the 34 patients with CKD. Therefore, Horzl shREmp of cine imaging may be more available than Vetrl Fraction of STIR for identifying myocardial tissue changes associated with CKD. Recently, Zhou et al. ${ }^{17}$ have shown that texture analysis of T1 mapping is more feasible than T1mapping in itself for assessing uremic cardiomyopathy, whereas the advantages of texture analysis over T1T2 mapping may include no additional imaging sequence and relatively facile assessment of the entire myocardium. The software used in this study is open access.

This study had some limitations. First, this is a single center study with a relatively small sample size. Second, endomyocardial biopsy was not performed in any patients, because of concerns about sample errors and complications, and because it lacks established indication for cardiomyopathy associated with CKD. Third, clinical relevance of T1T2 mapping or texture analysis was not determined in this study. Previous studies indicate that extracellular volume fraction is predictive for cardiovascular events associated with $\mathrm{CKD}^{8}$. Steady-state free precession imaging with $\mathrm{T} 2 / \mathrm{T} 1$ contrast not only provides cardiac functional parameters, but also reflects some histological characteristics that can be analyzed by texture analysis ${ }^{25,26}$. Aoki et al. ${ }^{4}$ reports that myocardial fibrosis, but not myocyte hypertrophy and disarray, is related to cardiac death in patients who undergo hemodialysis. Further study should be 
performed to assess the relationship between myocardial T1T2 values or texture features and prognosis of the patients with CKD.

In conclusion, both T1T2 mapping and texture analysis of T2-weighted STIR or cine images identifies myocardial tissue changes associated with CKD. Because cine imaging provides cardiac functional parameters and is insensitive to image artifacts, texture analysis for the cine images may be feasible for assessing myocardial tissue changes in the patients with CKD. T1T2 mapping is also feasible for this purpose.

\section{Materials And Methods Study Participants.}

Between August 2017 and February 2021, 34 consecutive patients with CKD were referred for cardiac MRI examinations to identify cardiac function and myocardial tissue changes. They were 25 men and 9 women ranging inage from 31 to 89 years (mean age, 67.1 years). There were 18 patients $(52.9 \%)$ with hypertension and $14(41.2 \%)$ with diabetes mellitus. Dialysis was given to 5 of 34 patients $(14.7 \%)$. In addition, $8(23.5 \%), 3(8.8 \%)$, and $1(2.9 \%)$ patient had heart failure, coronary artery diseases, and cardiopulmonary arrest, respectively. For comparison, 10 controls ( 5 men and 5 women; mean age, 41.1 years; age range, 21-78 years) were enrolled: their electrocardiogram or echocardiography was borderline positive, but cardiac MRI revealed no functional abnormalities and their myocardial T1 and T2 values were within the normal range ${ }^{14}$. LGE MRI showed no myocardial scar in the controls, while LGE MRI was not performed in any CKD patients because of their renal functional impairment. This retrospective, crosssectional study was approved by our institutional review board, and informed consent was given by all the patients and controls. All methods were performed in accordance with the relevant guidelines and regulation.

\section{Magnetic Resonance Imaging.}

All MRI examinations were performed using a 1.5 T system (Ingenia, Philips Healthcare, Best, the Netherlands). Breath-hold black-blood T2-weighted STIR was performed with the typical parameters as follows: repetition time (TR), 2 RR; effective echo time (TE), $80 \mathrm{~ms}$; field of view (FOV), $320 \times 320 \mathrm{~mm}^{2}$, imaging matrix, $220 \times 138$; and slice thickness, $10 \mathrm{~mm}$. Cine SSFP imaging was acquired with the imaging parameters as follows: TR, $3.2 \mathrm{~ms}$; TE, $1.6 \mathrm{~ms}$; FOV, 350 × $350 \mathrm{~mm}^{2}$, imaging matrix, 192 x 170; and slice thickness, $8 \mathrm{~mm}$. T1 mapping was performed with a $5 \mathrm{~s}(3 \mathrm{~s}) 3 \mathrm{~s}$ modified Look-Locker inversion recovery sequence acquired with a single-shot SSFP readout ${ }^{27}$. Therefore, 7 to 11 multicontrast images were acquired during 11-s breath-holding in short-axis midventricular slices. The imaging parameters for T1 mapping were as follows: TR, $2.7 \mathrm{~ms}$; TE, $1.3 \mathrm{~ms}$; flip angle, 35 ; FOV, 300 ' $300 \mathrm{~mm}^{2}$; imaging matrix, 152 ' 150; and slice thickness, $10 \mathrm{~mm}$. The delay time was $159.5 \mathrm{~ms}$ with an inversion time increment of 350 ms. T2 mapping imaging was performed with multiecho gradient- and spin-echo (GRASE) 
sequences $^{28}$. The imaging parameters for GRASE were as follows: TR, 1 RR; effective TE, 7.7-69.2 ms with 9 echoes and an echo train length of 5 ; flip angle, $35^{\circ}$; FOV, 300 ' $300 \mathrm{~mm}^{2}$; imaging matrix, $152^{\text {' }}$ 145; and slice thickness, $10 \mathrm{~mm}$.

\section{Image Analysis.}

Color-coded T1 and T2 maps were automatically generated on the operator console after T1T2 mapping scanning, and a team of three MR-dedicated radiological technologists measured T1T2 values of the septal myocardium according to the previous study ${ }^{10,29}$. Texture analysis was performed by a radiologist with 26 years of experience in cardiac MRI using the open-access software (MaZda; Institute of Electronics, Technical University of Lodz, Poland) ${ }^{23,27,30}$. The intensity range of the image under texture analysis was normalized by the default method: the intensity changes of the image ranged from 1 to 256 and 6 bits per pixel were used for image quantization. The entire left ventricular myocardium was enclosed manually, and a texture parameter calculation was conducted for T2-weighted STIR and cine images. The feature reduction methods, including Fisher coefficients, classification error probability, average correlation coefficients, and mutual information measure, were used to select the most discriminating features between the patients with CKD and controls.

\section{Statistical Analysis.}

The differences in the T1T2 values and texture features selected by the feature reduction methods were assessed between the patients with CKD and controls. An unpaired $t$ test was used to evaluate the differences, and $P<0.05$ was defined as significant. ROC analysis was used to determine the cutoff value for each analysis. The AUC was compared between the analyses and $P<0.05$ was defined as significant.

\section{Ethical approval.}

Voluntary informed consent was obtained from all subjects prior to their participation. The protocol was approved by the Nihon University Hospital Institutional Review Board.

\section{Declarations}

\section{Availability of Data}

All data will be made available from the corresponding author upon reasonable request.

\section{Author Contributions}


Research idea and study design: Y.A. data acquisition: Y.A., Y.O., C.A., N.S., Y.S. data analysis/interpretation: Y.A., Y.O., M.A., Y.S., N.M. statistical analysis: Y.A., M.A. Each author contributed important intellectual content during manuscript drafting and accepts accountability for the overall work by ensuring that questions pertaining to the accuracy or integrity of any portion of the work are appropriately investigated and resolved.

\section{Competing Interests:}

The authors declare no competing interest.

\section{References}

1. Go, A. S., Chertow, G. M., Fan, D., McCulloch, C. E. \& Hsu, C. Chronic kidney disease and the risks of death, cardiovascular events, and hospitalization. N. Engl. J. Med, 351, 1296-1305 (2004).

2. Foley, R. N. et al. Chronic kidney disease and the risks for cardiovascular disease, renal replacement, and death in the United States Medicare Population, 1998 to 1999. J. Am. Soc. Nephrol, 16, 489-495 (2005).

3. Silverberg, D., Wexler, D., Schwartz, B. M., laina, A. \& D. \& The association between congestive heart failure and chronic renal disease. Curr. Opin. Nephrol. Hypert, 13, 163-170 (2004).

4. Aoki, J. et al. Clinical and pathologic characteristics of dilated cardiomyopathy in hemodialysis patients. Kidney Int, 67, 333-340 (2005).

5. Wanner, C., Amann, K. \& Shoji, T. The heart and vascular system in dialysis., 388, 276-284 (2016).

6. Mark, P. B. et al. Redefinition of uremic cardiomyopathy by contrast-enhanced cardiac magnetic resonance imaging. Kidney Int, 69, 1839-1845 (2006).

7. Schietinger, B. J. et al. Patterns of late gadolinium enhancement in chronic hemodialysis patients. JACC. Cardiovasc. Imaging, 1, 450-456 (2008).

8. Xu, H. et al. Prognostic value of heart failure in hemodialysis-dependent end-stage renal disease patients with myocardial fibrosis quantification by extracellular volume on cardiac magnetic resonance imaging. BMC Cardiovasc Dis, 20, 12 (2020).

9. Graham-Brown, M. P. M. et al. Novel cardiac nuclear magnetic resonance method for noninvasive assessment of myocardial fibrosis in hemodialysis patients. Kidney Int, 90, 835-844 (2006).

10. Graham-Brown, M. P. et al. Defining myocardial fibrosis in haemodialysis patients with non-contrast cardiac magnetic resonance. BMC Cardiovasc Dis, 18, 145 (2018).

11. Stromp, T. A. et al. Quantitative gadolinium-free cardiac fibrosis imaging in end stage renal disease patients reveals a longitudinal correlation with structural and functional decline. Sci. Rep, 8, 16972 (2018).

12. Arcari, L. et al. Native T1 and T2 provide distinctive signatures in hypertrophic cardiac conditions comparison of uremic, hypertensive and hypertrophic cardiomyopathy. Int. J. Cardiol, 306, 102-108 
(2020).

13. Rutherford, E. et al. Myocardial changes in incident haemodialysis patients over 6-months: an observational cardiac magnetic resonance imaging study. Sci. Rep, 7, 13976 (2017).

14. Baessler, B. et al. Subacute and chronic left ventricular myocardial scar: accuracy of texture analysis on nonenhanced cine MR images., 286, 103-112 (2018).

15. Gould, J. et al. Mean entropy predicts implantable cardioverter-defibrillator therapy using cardiac magnetic resonance texture analysis of scar heterogeneity. Heart Rhythm, 16, 1242-1250 (2019).

16. Amano, Y. et al. Detection of myocardial tissue alterations in hypertrophic cardiomyopathy using texture analysis of T2-weighted short inversion time inversion recovery magnetic resonance imaging. J. Comput. Assist. Tomogr, 44, 341-345 (2020).

17. Zhou, H. et al. Texture analysis of native $T 1$ images as a novel marker for noninvasive assessment of uremic cardiomyopathy. J Magn Reson Imaging, 54, 290-300 (2021).

18. Bull, S. et al. Human non-contrast T1 values and correlation with histology in diffuse fibrosis., 99, 932-937 (2013).

19. Mewton, N. et al. Assessment of myocardial fibrosis with cardiovascular magnetic resonance. J. Am. Coll. Cardiol, 57, 891-903 (2011).

20. Liu, J. M. et al. Measurement of myocardial native T1 in cardiovascular diseases and norm in 1291 subjects. J. Cardiovasc. Magn. Reson, 19, 74 (2017).

21. Cao, Y. et al. Increased myocardial extracellular volume assessed by cardiovascular magnetic resonance T1 mapping and its determinants in type 2 diabetes mellitus patients with normal myocardial systolic strain. Cardiovasc. Diabetol, 17, 7 (2018).

22. Wang, L. et al. Myocardial T1rho mapping of patients with end-stage renal disease and its comparison with T1 mapping and T2 mapping: a feasibility and reproducibility study. J. Magn. Reason. Imaging, 44, 723-731 (2016).

23. Szczypinski, P. M., Strzelecki, M., Materka, A. \& Klepaczko, A. "MaZda” - a software package for image texture analysis. Comput. Methods. Prog. Biomed, 94, 66-76 (2009).

24. Zhang, H. et al. GPU-accelerated GLRLM algorithm for feature extraction of MRI. Sci. Rep, 9, 10883 (2019).

25. Bieri, O. \& Scheffler, S. Fundamentals of balanced steady state free precession MRI. J. Magn. Reson. Imaging, 38, 2-11 (2013).

26. Baessler, B. et al. Subacute and chronic left ventricular myocardial scar: accuracy of texture analysis on nonenhanced cine MR images. Radiology 286, 103 - 12(2018).

27. Amano, Y. et al. Relationship between measurement errors in myocardial T1 mapping and heart rate. Magn. Reson. Med. Sci, 19, 345-350 (2020).

28. Sprinkart, A. M. et al. Gradient spin echo (GRASE) imaging for fast myocardial T2 mapping. J. Cardiovasc. Magn. Reson, 17, 12 (2015). 
29. Rogers, T. et al. Standardization of T1 measurements with MOLLI in differentiation between health and disease - the Concept study. J. Cardiovasc. Magn. Reson, 15, 78 (2013).

30. Wu, Z. et al. Hepatitis $\mathrm{C}$ related chronic liver cirrhosis: feasibility of texture analysis of MR images for classification of fibrosis stage and necroinflammatory activity grade. PLoS One, 10, e0118297 (2015).

\section{Table}

Due to technical limitations, table 1 is only available as a download in the Supplemental Files section.

\section{Figures}




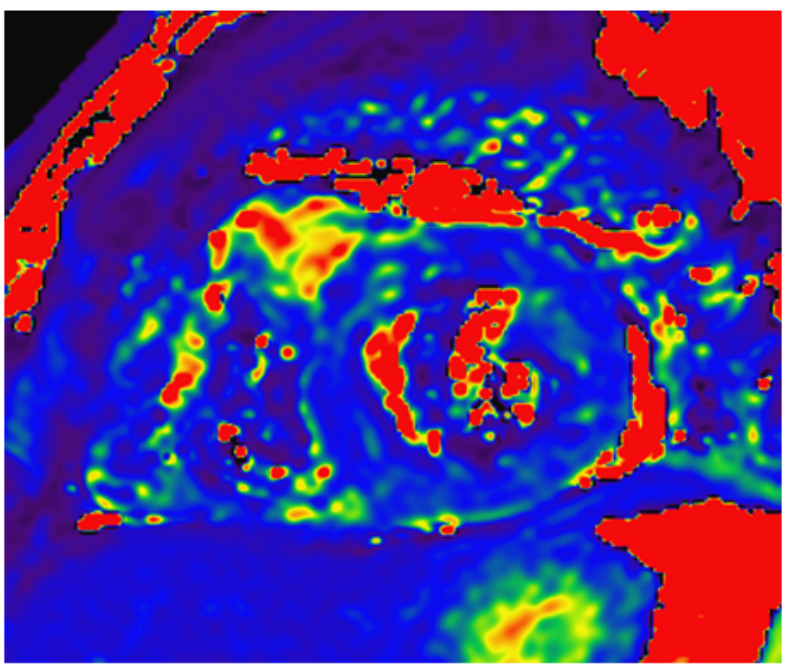

$\mathrm{C}$

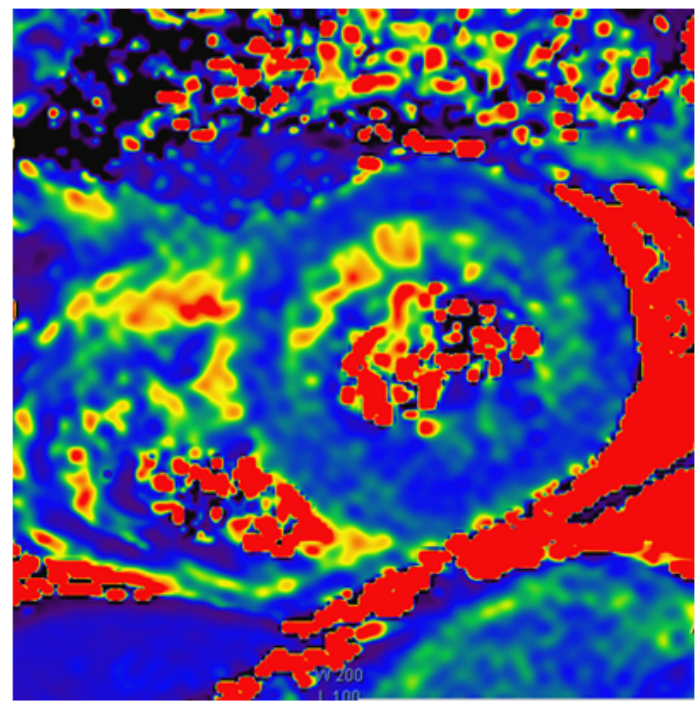

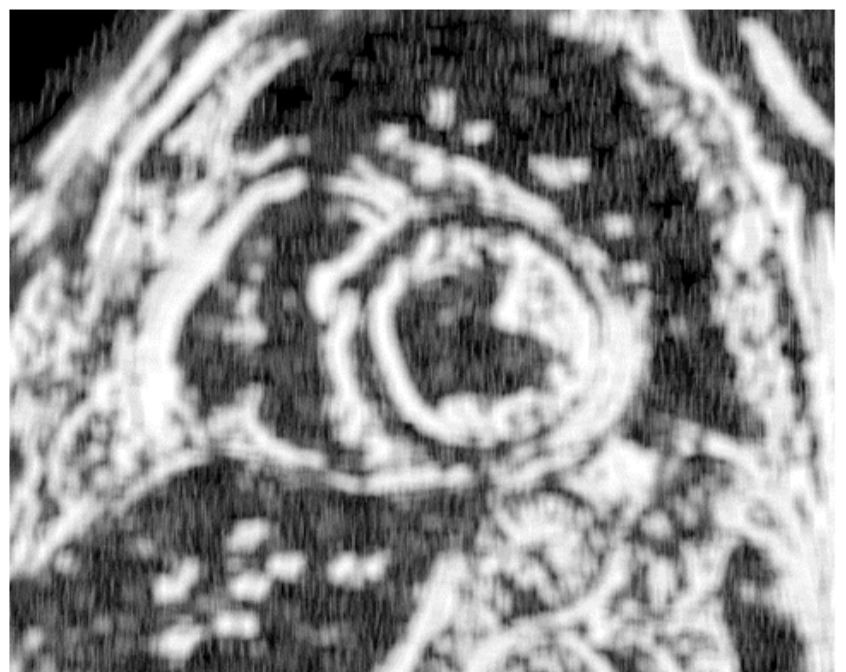

$\mathrm{D}$

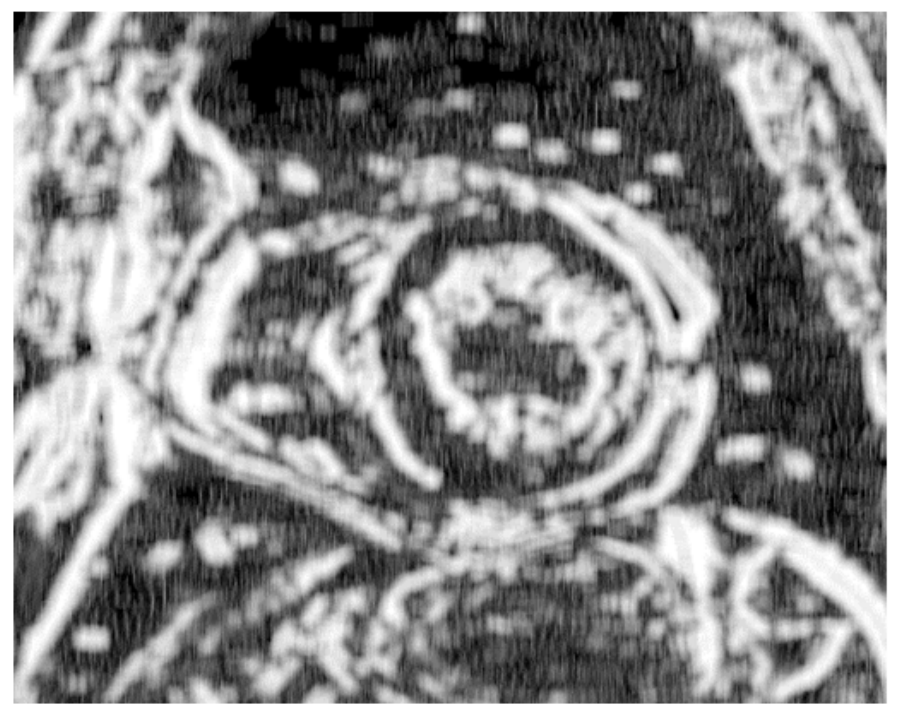

\section{Figure 1}

Panels A and B show T2 mapping and geometry of Horzl shREmp for cine imaging of a 61-year-old male control, respectively. The T2 value of the septal myocardium is $48.0 \mathrm{~ms}$ and Horzl shREmp is 0.642 . Panels C and D show T2 mapping and geometry of Horzl shREmp for cine imaging of a 71-year-old male patient with chronic kidney disease, respectively. The T2 value of the septal myocardium is $60.5 \mathrm{~ms}$ and Horzl shREmp is 0.492 in this case. The T2 value is prolonged and Horzl shREmp is smaller in the patient. 


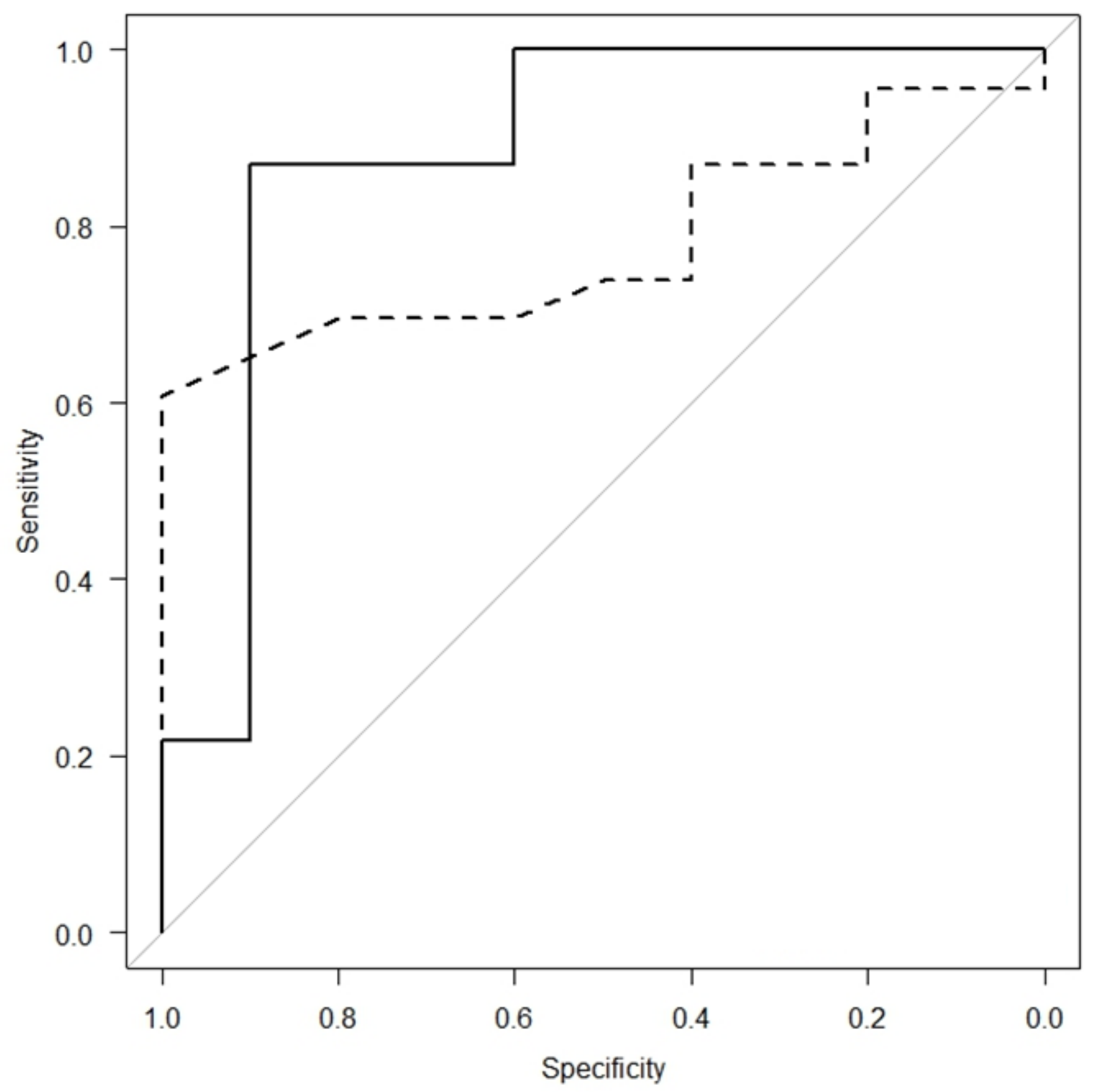

Figure 2

ROC curves of T2 mapping and Horzl shREmp for cine imaging to discriminate between controls and patients with chronic kidney disease. The AUC of Horzl shREmp is greater than that of T2 values $(0.883$ vs. 0.760$)$, but the differences are not significant $(P=0.36)$.

\section{Supplementary Files}

This is a list of supplementary files associated with this preprint. Click to download. 
- texturetable.docx

Page $12 / 12$ 